Review

\title{
No Pasaran! Role of the axon initial segment in the regulation of protein transport and the maintenance of axonal identity
}

\author{
Christophe Leterrier*, Bénédicte Dargent \\ Aix Marseille Université, CNRS, CRN2M UMR7286, 13344 Marseille Cedex 15, France
}

\section{A R T I C L E I N F O}

\section{Article history:}

Available online $\mathrm{xxx}$

\section{Keywords:}

Axon initial segment

Neuronal polarity

Neuronal transport

Protein traffic

Diffusion barrier

Ankyrin G

Cytoskeleton

\begin{abstract}
A B S T R A C T
The transmission of information in the brain depends on the highly polarized architecture of neurons. A number of cellular transport processes support this organization, including active targeting of proteins and passive corralling between compartments. The axon initial segment (AIS), which separates the somatodendritic and axonal compartments, has a key role in neuronal physiology, as both the initiation site of action potentials and the gatekeeper of the axonal arborization. Over the years, the AIS main components and their interactions have been progressively unraveled, as well as their role in the AIS assembly and maintenance. Two mechanisms have been shown to contribute to the regulation of protein transport at the AIS: a surface diffusion barrier and an intracellular traffic filter. However, a molecular understanding of these processes is still lacking. In the view of recent results on the AIS cytoskeleton structure, we will discuss how a better knowledge of the AIS architecture can help understanding its role in the regulation of protein transport and the maintenance of axonal identity.
\end{abstract}

(C) 2013 Elsevier Ltd. All rights reserved.

\section{Contents}

1. Introduction

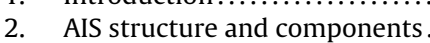

2.1. Ankyrin, spectrin and actin...

2.2. Membrane-partners of ankG ....

2.3. Regulated component interactions at the AIS .

2.4. Link between ankG and microtubules ...

3. AIS assembly and maintenance. ...

3.1. AIS assembly.....

3.2. AIS maintenance......

4. Traffic and targeting of AIS components

4.1. Traffic of intracellular AIS components

4.2. Targeting mechanisms of AIS membrane proteins

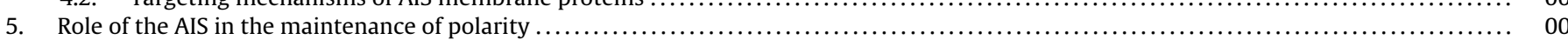

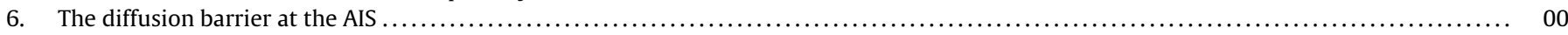

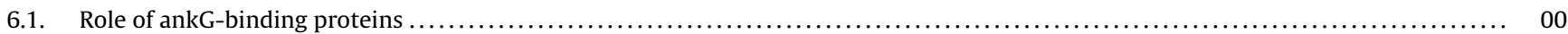

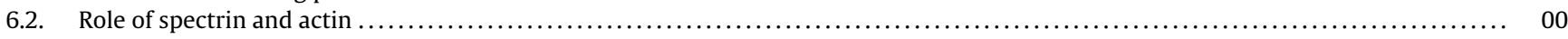

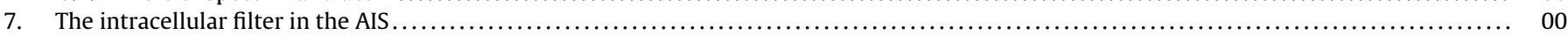

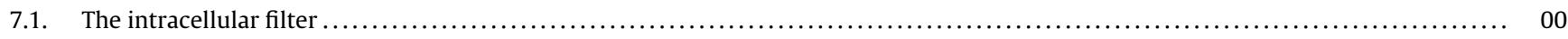

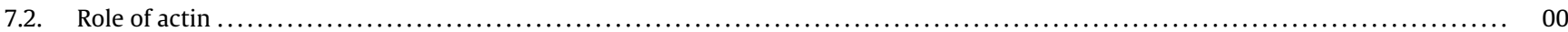

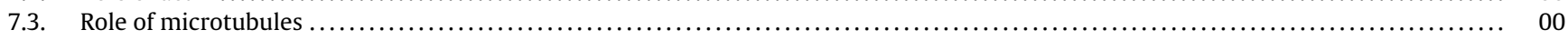

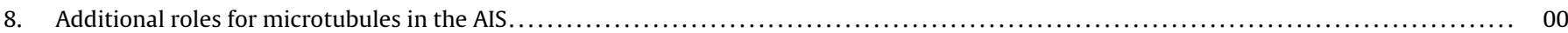

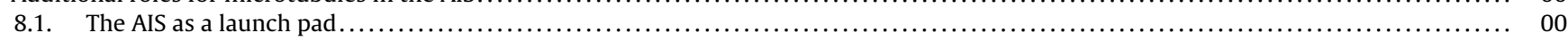

\footnotetext{
* Corresponding author at: Faculté de Médecine - Secteur Nord, Aix-Marseille Université CS80011, Bd Pierre Dramard, 13344 Marseille Cedex 15, France. Tel.: +33 4916989 30; fax: +33491090506.

E-mail address: christophe.leterrier@univ-amu.fr (C. Leterrier).
}

1084-9521/\$ - see front matter @ 2013 Elsevier Ltd. All rights reserved. http://dx.doi.org/10.1016/j.semcdb.2013.11.001 


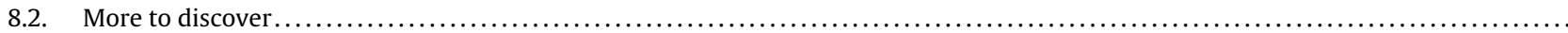

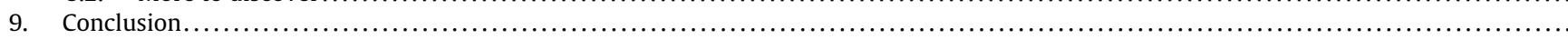

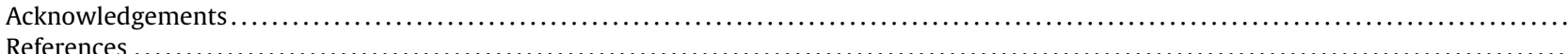

\section{Introduction}

The directionality of signal transmission in the brain is supported by the exquisite asymmetry of neurons. Neurons are divided in two main compartments that differ in their molecular composition and organization: the soma and dendrites that receive and integrate synaptic inputs (somatodendritic compartment), and the axon that sends the signals to downstream cells through an extensive arborization (axonal compartment, Fig. 1A). A number of studies have progressively uncovered how cellular polarity is established during neuronal development [1]. Once polarity is established, a sequential growth phase leads to the building of axonal and dendritic arborizations. Mature neurons then face the daunting task of maintaining the asymmetry of this intricate architecture for years in rodents, and decades in humans [2].

A key player for the maintenance of neuronal polarity lies between the somatodendritic and axonal compartments: the axon initial segment (AIS, Fig. 1A). Its localization along the first $20-40 \mu \mathrm{m}$ of the axon underpins its two main functions: the generation of the action potential, and the preservation of axonal identity [3]. This review will focus on how the AIS maintains neuronal polarity by regulating protein mobility and traffic between the soma and the axon. We first describe the AIS main components, how the AIS is assembled, and how its components are targeted and concentrated. Then, we review how the AIS controls protein transport in the cell via the formation of a surface diffusion barrier and an intracellular traffic filter. We finally discuss how the expanding repertoire of identified proteins and interaction at the AIS, together with recent results on the AIS cytoskeleton structure, spark new hypotheses on the molecular details of these processes.

\section{AIS structure and components}

\subsection{Ankyrin, spectrin and actin}

First described by Kölliker and Remak in the middle of the 19th century [4], the AIS morphology was characterized by the pioneers of electron microscopy in the 1960s [5]. They defined morphological criteria for identifying the AIS on brain electron microscopy images, including fascicles of microtubules, and a dense layer of material undercoating the plasma membrane. Like in the rest of the axon, AIS microtubules are uniformly oriented with plus-ends toward the distal axon. The AIS microtubule fascicles are clearly seen on transverse sections, where they appear as small rings connected by filaments (Fig. 1B).

The plasma membrane undercoat is composed of an ankyrin-spectrin scaffolding complex that defines the identity and functions of the AIS. The main component of the AIS scaffold is ankyrin G [6] (ankG, Fig. 1C and D). The mammalian ankyrin family is composed of three genes encoding for ankyrin $\mathrm{R}$
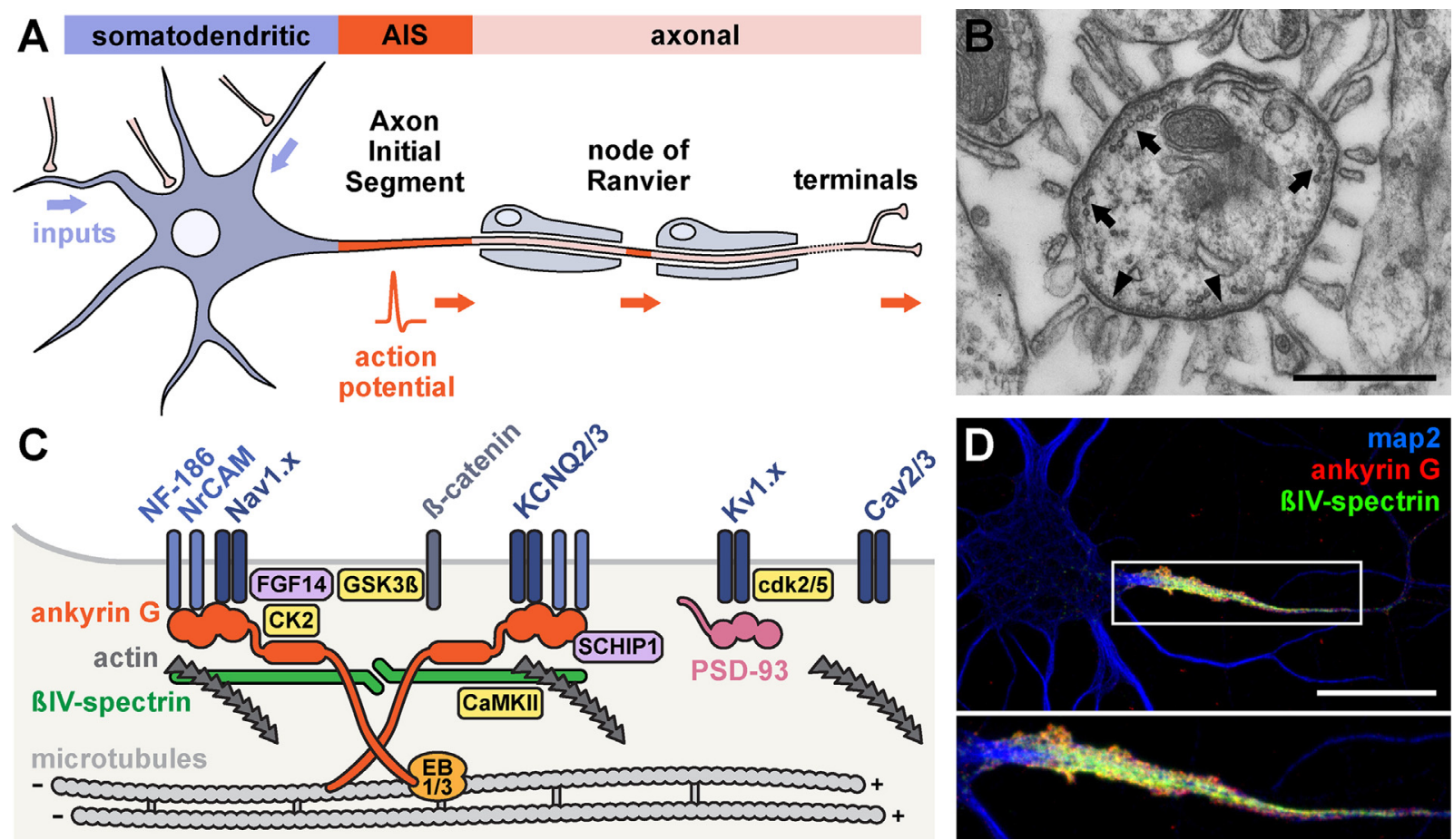

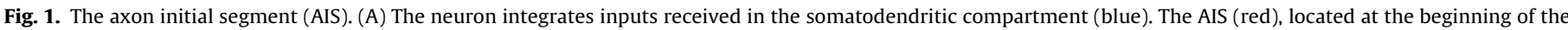

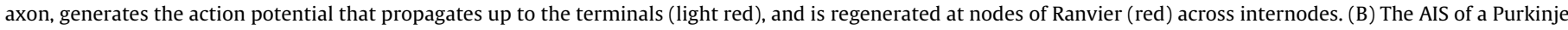

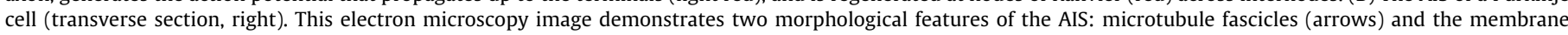

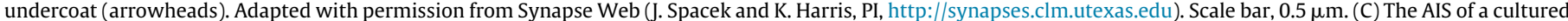

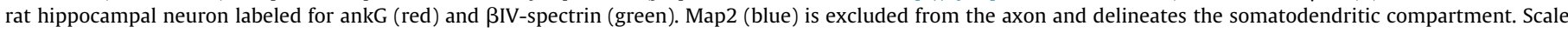

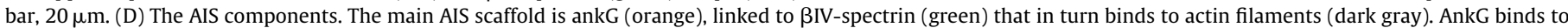

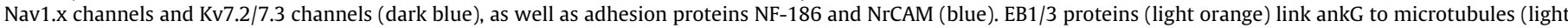

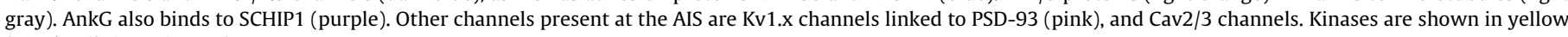
(see details in main text). 
(ankR, first identified in erythrocytes), ankyrin B (ankB, isolated from brain), and ankG (initially characterized as a component of nodes of Ranvier). Although the short isoforms of ankG are ubiquitous, the two long ankG isoforms of 270 and $480 \mathrm{kDa}$ are uniquely found in neurons, concentrated at the AIS and nodes of Ranvier membrane undercoat [7-9]. The longest isoform, ankG 480 , is a large 4400 amino acids protein composed of a membrane binding domain, a spectrin-binding domain, a serine-rich domain, a long tail (2200 aa), and a carboxy-terminal domain. The second long isoform of ankG found at the AIS, ankG 270, lacks the last 1900 amino-acids of the tail compared to ankG 480 [7]. The other large ankyrin expressed in neurons, ankB, is found as two 440 and $220 \mathrm{kDa}$ isoforms that are not concentrated in the AIS, but localize to the distal part of unmyelinated axons [6].

Similarly to the ankyrin-spectrin complex lining the erythrocyte membrane, ankG is bound via its spectrin-binding domain to a specific member of the spectrin family, identified as $\beta I V$-spectrin in the AIS [10] (Fig. 1C and D). Classically, spectrins are found as hetero-tetrameric complexes of two $\alpha$-spectrins and two $\beta$ spectrins, with $\beta$-spectrins bound to actin filaments [6]. Although $\alpha$ II-spectrin is found along the whole axon, a specific $\alpha$-spectrin has not yet been characterized at the AIS. It is thus unknown if AIS spectrins exist as classical $\alpha-\beta$ tetramers or as a different complex. The $\beta I V$-spectrin isoforms concentrated at the AIS are the $\Sigma 1$ and $\Sigma 6$ $\beta I V-s p e c t r i n s$. The $\beta I V \Sigma 6$ isoform is necessary for proper AIS organization $[11,12]$ although it lacks the actin-binding domain found on other isoforms, questioning whether this AIS spectrin complex is linked to actin. In line with this question, actin enrichment in the AIS has been reported in a handful of studies [13-15], but only detected after specific detergent extraction for others [16,17]. However, even with no concentration, specific actin organization occurs in the AIS [15], consistent with its major role in establishing the AIS mobility barrier (see below). Finally, ankG has been shown to interact with schwannomin-interacting protein SCHIP1, a protein that concentrates in the AIS, but whose function remains unknown [18].

\subsection{Membrane-partners of ankG}

AnkG also binds via its membrane-binding domain to membrane proteins that accumulate at the AIS (Fig. 1C). AnkG binds to voltage-gated sodium (Nav) channels [19] via a motif located in their intracellular loop between transmembrane domains II and III $[20,21]$. It leads to the concentration of Nav channels in the AIS compared to the soma, with an enrichment estimated between 3[22] and 40-fold [23,24], depending of the experimental approach. Nav channel accumulation underlies the role of the AIS in the final integration of somatodendritic inputs and the generation of the action potential in most neuronal types (reviewed in $[4,25]$ ). The main Nav channel types present in the AIS are Nav1.6 and Nav1.2, although Nav1.1 is also found at beginning of the AIS in certain neurons (reviewed in [26]). Additionally, a developmental switch occurs between Nav1.2 and Nav1.6 [27], as well as a proximodistal complementary distribution of Nav1.2 and Nav1.6 in mature neurons [28]. How different Nav channels bearing similar ankG binding site can be selectively enriched along the AIS is an interesting and still open question. Potassium channels Kv7.2 and Kv7.3 (KCNQ2/KCNQ3) share a binding site to ankG that is analogous to the Nav channel motif, and accumulate at the AIS as heteromers, where they dampen excitability [29]. Ion channels that do not possess an identified ankyrin-binding site have also been localized to the AIS. Potassium channels Kv1.1/1.2 localize at the distal AIS, bind to post-synaptic density protein PSD-93 [30,31], and can modulate AP initiation, as do Cav2/3 calcium channels present along the AIS [32].

AnkG also binds via its membrane-binding domain to cell adhesion molecules from the L1-CAM family [6]. Binding to ankG results in the AIS and nodal concentration of two family members, the $186 \mathrm{kDa}$ isoform of neurofascin (NF-186), and the neuronal cell adhesion molecule (NrCAM) [33]. Notably, a single ankG protein can simultaneously bind two CAMs and one ion channel [6]. NF186 has been shown to recruit components of the extracellular matrix such as brevican to the AIS of hippocampal neurons [34], and ankG-directed concentration of NF-186 is important for GABAergic innervation of the pinceau terminal around the Purkinje cells AIS [35].

\subsection{Regulated component interactions at the AIS}

Although it is a stable complex, the AIS is not static: a number of kinases regulate the interaction between AIS components. NF-186 and NrCAM can be phosphorylated at their cytoplasmic FIGQY motif, inhibiting their interaction with ankG [36]. Protein kinase CK2 phosphorylates the ankG binding site on Nav channels to strengthen their interaction with ankG [37], and participates in AIS formation [38]. Cyclin-dependent kinases cdk2 and cdk5 phosphorylate the $\operatorname{Kv} \beta$ subunit, regulating their surface expression in the distal AIS Kv complex [39], whereas cdk5 controls the length of an AIS-like compartment in Drosophila [40]. The $\mathrm{Ca}^{2+} /$ calmodulin dependent protein kinase CaMKII associates with $\beta I V-s p e c t r i n$ and phosphorylates Nav channels [41]. Finally, the glycogen synthase kinase GSK3 $\beta$ phosphorylates $\beta$-catenin in the AIS, regulating the maintenance of the AIS [42], and controls the interaction of Nav channels with fibroblast growth factor FGF14, participating in channels concentration in the AIS [43].

\subsection{Link between ankG and microtubules}

The large size of the ankG isoforms found at the AIS suggests that they could interact with intracellular targets. Potential partners for ankG inside the AIS are microtubules, as ankR and ankB bind microtubules in vitro, directly or indirectly $[44,45]$. Sobotzik et al. studied the AIS structure in Purkinje cells lacking ankG, thanks to a cerebellum-specific ankG knockout mouse [46]. They showed that the characteristic microtubule fascicles at the AIS are dispersed in the absence of ankG, suggesting interplay between ankG and microtubules [47]. Leterrier et al. thus searched for potential partners in this interaction and identified the end-binding proteins members EB1 and EB3 as links between microtubules and ankG [48]. EB proteins are usually associated with growing microtubule plus-ends, where they organize a complex implicated in interactions with the cellular cortex structures [49]. EB1 and EB3 interact with ankG, and are stabilized along the microtubule lattice in the AIS. This link between ankG and microtubules could have important implications for the filtering of intracellular transport [48] (see below).

\section{AIS assembly and maintenance}

\subsection{AIS assembly}

How is the large AIS macromolecular complex built and maintained? In neuronal cultures, all components assemble in quick succession between 3 and 4 days in culture [34,50]. In vivo studies of mice lacking ankG in Purkinje cells [35,46,51], together with knockdown of AIS components in developing neurons [34] have demonstrated that ankG is necessary for the recruitment of other AIS components: Nav channels, $\beta$ IV-spectrin, NF-186 and NrCAM. Conversely, the ankG-binding motif of Nav channels [20,21,52], Kv7 channels [29,53], NF-186 [50] and $\beta I V-s p e c t r i n ~[54]$ is necessary for their concentration in the AIS. Interestingly, Nav channel knockdown can prevent formation of the AIS in motoneurons, suggesting that they could also play a role in AIS assembly for certain neuronal 
types [55]. The ankG-dependent "inside-out" AIS formation contrasts with the "outside-in" assembly of nodes of Ranvier, where extracellular glial signals first bind to NF-186, which recruits ankG, allowing Nav channel concentration [56-58]. If ankG recruits all other components to the AIS, how is ankG is recruited to the AIS in the first place? The Rasband lab recently demonstrated the existence of an antagonistic mechanism between ankG and ankB [59]. After the initial specification of the axon, ankB is targeted to the distal axon and progressively fills the axon toward the soma. The later onset of ankG expression results in the distribution of ankG along the remaining proximal stretch of the axon that becomes the AIS [59]. In developing motoneurons, the early expression of ankG could lead to a different AIS formation pattern: ankG appears along the whole axon at embryonic day E9.5 and concentrates in the AIS after E11.5, ankB being present along the whole axon [9].

\subsection{AIS maintenance}

AnkG also has a very important role for AIS maintenance: depleting ankG in mature neurons results in AIS disassembly, with a dispersion of the accumulated $\beta$ IV-spectrin, ion channels and CAMs [60]. However, once assembled, the integrity of the AIS also depends on the presence of the other components. Long-term absence of $\beta I V$-spectrin in knockout mice decreases ankG and Nav channel accumulation in the AIS $[11,12,61]$. In vivo depletion of NF-186 in Purkinje cells results in a progressive disappearance of the AIS $[62,63]$. Finally, depletion of the microtubule-ankG link formed by EB1 or EB3 results in a partial disassembly of the AIS in cultured neurons [48]. These results suggest that although ankG is the primary organizer of the AIS, its components are ultimately interdependent.

\section{Traffic and targeting of AIS components}

\subsection{Traffic of intracellular AIS components}

Beyond the interactions and hierarchies between AIS components, how each component is targeted and delivered to the AIS remains elusive. How does ankG enter the axon before competing with ankB as proposed by Galiano et al. [59]? Several ankG domains, notably the serine-rich domain, seem to be important for its axonal targeting [64]. Palmitoylation of the ankG membrane binding domain, together with the linker before the spectrin-binding domain, are implicated in ankG submembrane localization in axons $[65,66]$. We know that ankG recruits its partners to the AIS, but whether it associates with any of them and is co-transported before arriving at the AIS remains an open question. In particular, this includes other AIS intracellular components such as $\beta I V-s p e c t r i n$ and $\mathrm{EB} 1 / 3$.

\subsection{Targeting mechanisms of AIS membrane proteins}

Two general mechanisms participate in the concentration of membrane proteins the AIS: selective endocytosis from the somatodendritic compartment, and capture by ankG. Using chimeras bearing various intracellular parts of Nav channels, the Dargent lab has shown that the C-terminus and the loop between transmembrane domains II and III target Nav channels to the axon. Interestingly, there is no direct targeting to the axon, but rather unpolarized export to the plasma membrane followed by selective endocytosis from the somatodendritic compartment $[67,68]$. It would be interesting to test whether axonal targeting of Kv7.2/7.3 heteromers, which occurs via motifs in the C-terminus domain [69], also depends on a selective endocytosis step. Interestingly, NF-186 also accumulates at the AIS after selective endocytosis from the somatodendritic compartment, in a doublecortin-dependent manner [70]. Once AIS membrane proteins have reached the axonal surface, binding to ankG is thought to drive their concentration at the AIS by capturing proteins that diffuse along the plasma membrane. It has been observed that Nav channel diffusion is blocked at the AIS $[13,71]$. Brachet et al. using Nav-Kv chimeras bearing mutants of the Nav ankyrin-binding domain, have demonstrated that channel immobilization in the AIS is caused by their interaction with ankG [72]. Binding to ankG is also able to slow down NF-186 diffusion [36], resulting in its immobilization at the AIS [50], and prevents NF-186 endocytosis mediated by doublecortin [70].

\section{Role of the AIS in the maintenance of polarity}

Once assembled, the AIS separates the axon from the rest of the neuron and thus helps maintaining neuronal polarity. First proposed after the discovery of the diffusion barrier between the soma and the axon $[71,73]$, the functional evidence confirming this hypothesis was provided only a few years ago. Hedstrom et al. used long-term depletion of ankG in cultured neurons, and showed that it not only induces disassembly of the AIS, but also leads to a progressive loss of axonal identity, with appearance of somatodendritic markers in the proximal axon [60]. These results were confirmed in vivo by examining the axonal morphology of mice lacking ankG in Purkinje cells. The proximal axon of these cells exhibits spines containing post-synaptic assemblies that appear gradually during maturation [47]. How is the AIS keeping somatodendritic proteins from invading the axon? Studies have shown that the AIS regulates protein transport between the soma and the axon through two mechanisms: a surface diffusion barrier, and an intracellular traffic filter (Fig. 2A and B).

\section{The diffusion barrier at the AIS}

Lipids and membrane proteins cannot freely diffuse through the AIS, but are slowed down or immobilized, resulting in the isolation of the somatic and axonal membrane composition (Fig. 2A). This diffusion barrier was first identified by Kobayashi et al., who discovered that fluorescent lipids cannot diffuse from the axon back to the soma [73], before being confirmed at the single molecule level by video-rate tracking of membrane lipids [74]. Restricted diffusion of axonal proteins at the AIS was first shown by experiments measuring tractability of L1 or Thy-1 attached beads [17] and confirmed by single particle tracking of proteins labeled with quantum dots $[14,72]$.

\subsection{Role of ankG-binding proteins}

The immobilization of lipids in the proximal axon is temporally correlated to the AIS formation [13]. Furthermore, disassembly of the AIS through ankG depletion leads to the disappearance of the diffusion barrier [14]. How does the AIS restrict the diffusion of membrane components? The anchored-proteins "pickets" model from the Kusumi lab proposes that a subset of membrane proteins interacts with the cytoskeletal elements underlying the membrane, resulting in rows of immobilized pickets that slow diffusion of membrane proteins and lipids [13]. In the AIS, the pickets would be the membrane proteins immobilized by their interaction with the ankG/spectrin undercoat: ion channels and CAMs [50,71,72]. Brachet et al. showed that ankG-binding proteins were immobilized as soon as ankG accumulated in the AIS, before the establishment of the diffusion barrier [72]. Thus, ankG-binding membrane proteins are recruited as a preliminary step, suggesting they are the pickets of the AIS diffusion barrier [13]. Interestingly, the phosphoregulation of channels and CAMs that modulate their binding to 
A

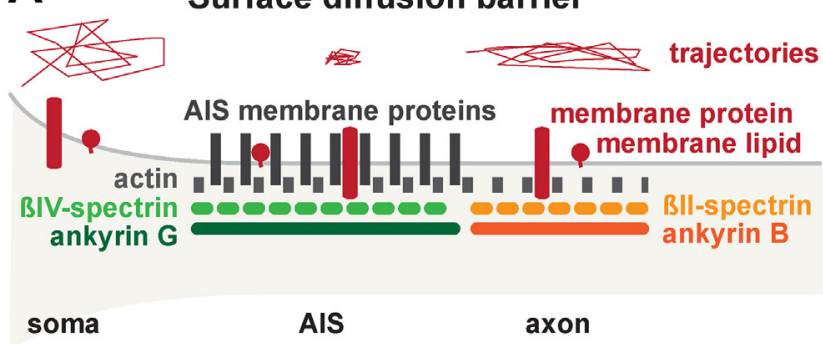

B

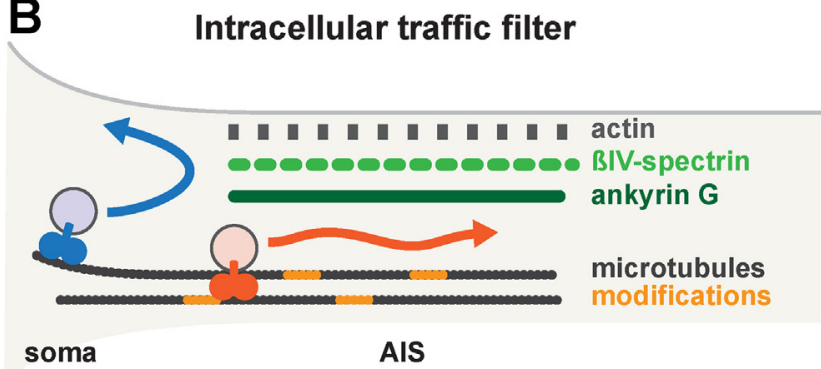

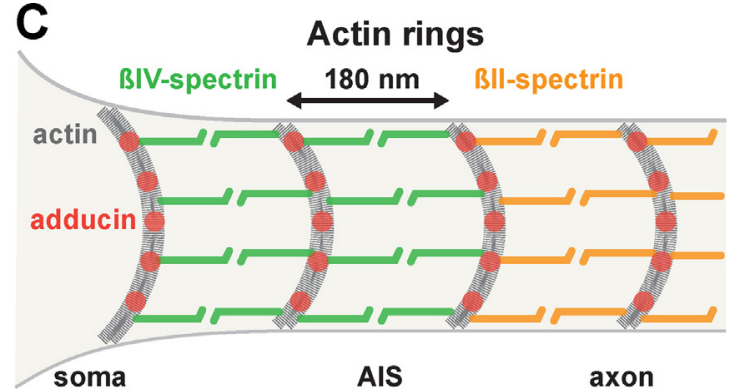

D

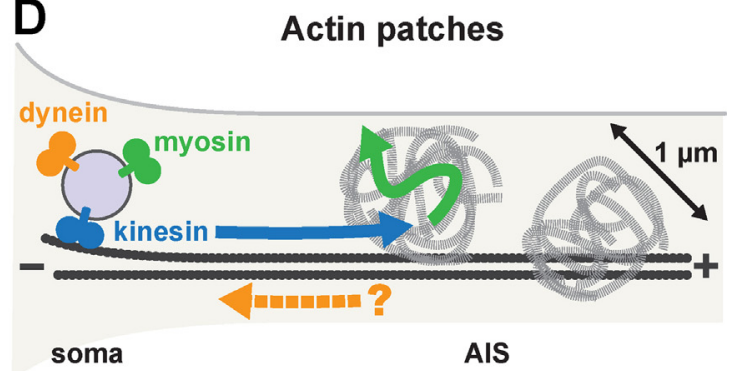

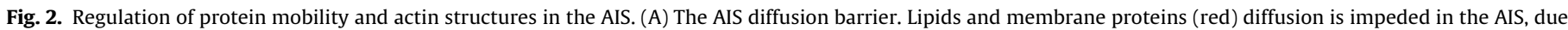

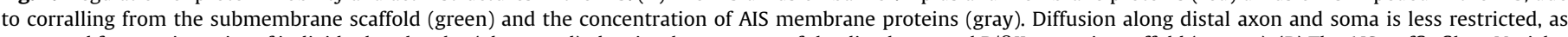

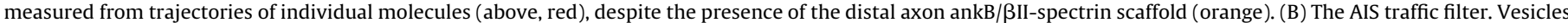

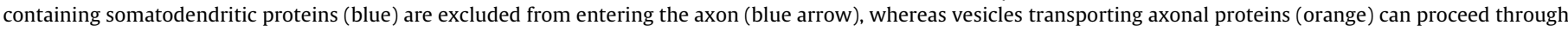

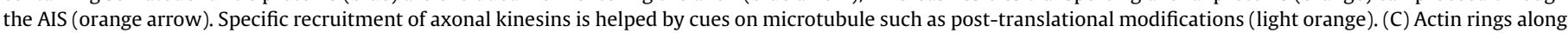

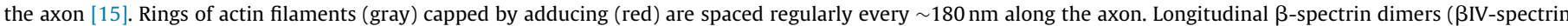

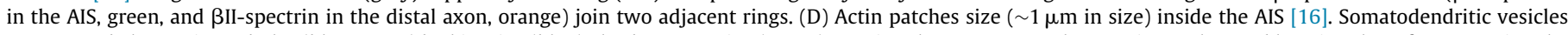

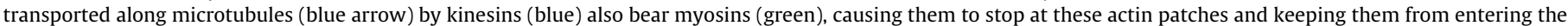
axon (green arrow). Also depicted is the hypothetical role of dynein in transporting non-axonal cargoes back to the soma (orange).

ankG also modify their diffusive properties [36,72], suggesting that the strength of the diffusion barrier could be dynamically regulated.

\subsection{Role of spectrin and actin}

Actin is necessary for the presence of the diffusion barrier, as actin filaments disruption leads to its disappearance [13,14,17]. $\beta I V-S p e c t r i n$ plays a role in restricting the diffusion of L1 in the AIS compared to the distal axon [75]. In erythrocytes, spectrin connects complexes of actin and ankyrin with a $\sim 100 \mathrm{~nm}$ hexagonal pattern that limits diffusion of membrane proteins [6]. At the AIS, what specific organization of the spectrin/actin cytoskeleton leads to the immobilization of membrane components? Recently, Xu et al. used super-resolution microscopy to reveal the localization of actin and spectrin in axons with a $\sim 20 \mathrm{~nm}$ resolution. They showed that actin is forming ring-like structures spaced $\sim 180 \mathrm{~nm}$ apart around the circumference of the axons. The middle part of spectrin tetramers is precisely located between the actin rings, resulting in a complementary bands pattern. This suggests that spectrins lie along the plasma membrane, connecting successive actin rings [15] (Fig. 2C). This organization is strikingly reminiscent of the periodic annular structure previously observed in electron microscopy images of the AIS undercoat [76].

Do these successive actin/spectrin hurdles cause the AIS diffusion barrier? The actin rings are detected along the whole axon, linked by $\beta I V$-spectrin in the AIS and by $\beta$ II-spectrin in the distal axon [15] (Fig. 2C). Thus, the presence of these rings cannot explain the diffusive properties of the AIS compared to the distal axon. The diffusion barrier is likely caused by additional AIS proteins associated to these structures, such as the ion channels and CAMs acting as pickets. It would nevertheless be interesting to extend these findings in relation to the known AIS structure. Is there a difference in the arrangements of the two AIS $\beta I V-s p e c t r i n$ isoforms, given that one of them lacks an actin-binding domain? Is ankG also organized in ring-like structures? How are the actin/spectrin rings assembled during development, given that ankG recruits $\beta I V$-spectrin during AIS formation [54]? Interestingly, impairment of the AIS structure has been reported after actin filaments disruption in young neurons $[14,55]$, whereas AIS of older neurons do not seem to be affected $[38,77]$.

\section{The intracellular filter in the AIS}

\subsection{The intracellular filter}

Besides the surface diffusion barrier, the AIS participates in the maintenance of axonal identity by regulating the intracellular traffic. Live-cell imaging of GFP-fused proteins shows that vesicles transporting axonal proteins can enter in both axon and dendrites, whereas those containing somatodendritic proteins are excluded from entering the axon [78,79]. The intracellular diffusion of large molecules and vesicular transport toward the axon is significantly slowed at the AIS, demonstrating the existence of an AIS transport filter [14]. Importantly, this filter acts like a sieve, as the entry of macromolecules depends on their size, and the passage of vesicles depends on the processivity of the motor proteins propelling them (Fig. 2B). This filter is established at the same time as the AIS (between 3 and 5 days in culture), and depends on AIS integrity, as shown by its disappearance after ankG depletion [14].

\subsection{Role of actin}

Disruption of actin filaments impairs the AIS filter, allowing the entry of large macromolecules and somatodendritic vesicles into the axon $[14,80]$. How can actin regulate intracellular traffic in the AIS? Lewis et al. have shown that myosin $\mathrm{V}$ binding can target membrane proteins to the somatodendritic compartment [80]. Subsequent work showed that myosin VI, which travels 
toward the opposite side of actin filaments, can conversely participate in the targeting of axonal protein [81]. This led the authors to propose that the AIS contains actin filaments, which stop somatodendritic vesicles entering the axon via myosin $\mathrm{V}$-based interaction and may bring them back to the soma [79]. Strikingly, scanning electron microscopy images of detergent-extracted cells showed an accumulation of actin into $\sim 1 \mu \mathrm{m}$ globular clusters the AIS. Somatodendritic vesicles were often stopped at these clusters [16], supporting a role of actin as a selective road block in the AIS (Figure 2D). It is not clear if these clusters could mediate selective retrieval of vesicles toward the soma, and they are clearly different from the rings observed by $\mathrm{Xu}$ et al. [15]. Observing the fine structure of actin in the AIS of living cells, for example using photoactivated localization microscopy (PALM) could help to conciliate these findings. Coupled with imaging of vesicular cargoes, PALM of actin could further clarify the role of actin in filtering transport through the AIS.

\subsection{Role of microtubules}

Recent results suggest that microtubules, besides supporting vesicular transport, could play a role in the AIS sieve assembly. Acute microtubule disruption or stabilization does not disassemble the AIS of mature neurons [26,77,82]. However, microtubules are necessary for blocking retrograde diffusion of the microtubuleassociated protein Tau from the axon to the soma [77]. The discovery of an interaction between ankG and microtubules via EB proteins [48] revigorates the hypothesis that the large ankG isoforms could stretch across the AIS between the plasma membrane and the cytoplasm, and connect the undercoat complex to microtubules [33]. The ankG tail is flexible and unstructured, and could stretch up to $0.5 \mu \mathrm{m}$ in length if fully extended in the case of the longest isoform [6]. This could explain how the AIS scaffold regulates traffic toward the axon: an accumulation of ankG tethers between membrane protein partners and microtubules would form a sieve and impede entry of large macromolecules and weakly powered vesicles into the axon [14].

This physical link between membrane proteins and microtubules via ankG, and its relevance for filtering traffic through the AIS, has yet to be tested. Importantly, the details of an interaction between ankG and microtubules are still largely unknown. Although it is known that ankG binds to the hydrophobic pocket of EBs [26], what part of ankG binds to EBs is not. The large ankG 480 contains a putative SxIP EB-binding motif in its tail [83], but it does not seem to be present in the shorter ankG 270. Besides EBs, it is likely that ankG can bind microtubules directly or via other yet unidentified partners. Antibodies reacting with microtubule-associated epitopes in the AIS suggest that unknown microtubule-bound AIS components remain to be discovered $[82,84]$. Interestingly, a recent study found that the ankyrin repeats of the Drosophila no mechanoreceptor potential C(NOMPC) channel are bound to microtubules, this membrane-microtubule tethering resulting in the mechanosensitive properties of the channel [85]. Combining the details of the ankG-microtubule linkage and the ankG interaction with the spectrin rings discovered by $\mathrm{Xu}$ et al. [15] would help testing a molecular model where the AIS traffic filter is formed by an accumulation of ankG tethers across the axon.

\section{Additional roles for microtubules in the AIS}

\subsection{The AIS as a launch pad}

A growing body of evidence suggests that microtubules contribute to axonal cargoes steering via specific modifications and selective recruitment of kinesins [2]. Kinesins are microtubuleassociated motors that drive transport of organelles and vesicular cargoes. Most kinesins travel toward the microtubule plus ends, resulting in anterograde transport in axons, which contain uniform plus end-out microtubules [86]. The kinesin KIF5/kinesin-1 has been shown to transport several axonal cargoes, and also a few somatodendritic ones [86]. KIF5, when expressed as a motor head fragment, is selectively targeted to axons, and a nonprocessive KIF5 head rigor mutant accumulates selectively along the AIS microtubules, suggesting that these microtubules harbor a specific cue for KIF5 recruitment [74]. What are the signals on AIS microtubules that are recognized by the KIF5 motor head? AIS microtubules are enriched in post-translational modifications (PTMs) typical of stable microtubules such as acetylation, detyrosination and polyglutamylation [87-89]. As KIF5 preferably binds to PTM-rich, stabilized microtubules $[90,91]$, it was proposed that PTMs direct the recruitment of KIF5 to the AIS. However, reports differ on whereas detyrosination [87], acetylation [89,90] or a combination of multiple PTMs [88] have a role for KIF5 preferential recruitment. Alternative candidates for microtubule cues are GTP remnants along the AIS microtubules. A microtubule typically has tubulin monomers bound to GTP at the growing plus-end, and to GDP along the shaft, reflecting progressive hydrolysis of GTP. But microtubules can also contain small stretches of non-hydrolyzed GTP along their length that are called GTP remnants [92]. Nakata et al. showed that these remnants are enriched along the axonal microtubules, and play a role in recruiting KIF5 toward the axon [93]. Moreover, as the plus-end GTP cap is also the preferred location of EB proteins interactions, one could speculate that these GTP remnants have a role in the enrichment of EBs at the AIS, allowing them to link the microtubule shaft to ankG [48].

\subsection{More to discover}

Results in model organisms suggest that key mechanisms may be still missing in our understanding of polarized targeting. Dynein is a molecular complex that moves along microtubules toward their minus end, and drives retrograde transport in axons [2]. In mammalian neurons, dynein has a role in targeting proteins to dendrites via transport along the minus end-out microtubules found in proximal dendrites, where microtubule orientation is mixed [94]. Furthermore, dynein contributes to the polarized organization of the Golgi apparatus [95], and organizes axonal microtubules in Drosophila neurons [96]. One could hypothesize that dynein also excludes somatodendritic cargoes from the axon by bringing them back to the soma along AIS microtubules, similarly to what was proposed for myosin $\mathrm{V}$ along actin filaments [80]. In the nematode Caenorhabditis elegans, the ankyrin homolog unc-44 allows the axonal targeting of unc-33/CRMP (collapsin response mediator protein), the establishment of uniform microtubule polarity, and the proper targeting of unc-116/KIF5 to the axon [97]. Finally, the C. elegans unc-16/Sunday Driver/JIP3 (JNK interacting protein), an adaptor that interacts with both kinesin and dynein, is present in the proximal axon and necessary for regulating organelle entry into the axon [98]. Altogether, these results suggest that further interplay between the AIS scaffold, microtubules and motor proteins may exist and could play a crucial role in shaping polarized traffic and axonal identity.

\section{Conclusion}

The AIS is now recognized as a key compartment in neuronal physiology. It is instructive to draw a parallel with another neuronal structure that has been thoroughly studied in the past decades: the neuronal synapse. Both structures are characterized by an 
accumulation of channels and adhesion proteins, controlled by interactions with specialized scaffolds. However, at the level of the cell, the synapse is an endpoint, whereas the AIS is a gate between the neuron's main polarized domains. This underlies its role in the organization of the cell and in the maintenance of polarity. Multiple components may be still missing in our view of the AIS architecture, and how components are arranged is largely unknown. Deciphering the fine structure of the AIS will allow understanding how the AIS shapes intracellular traffic and maintains axonal identity. Moreover, similar to the synapse, the AIS macromolecular complex is not a static assembly. Recent results have shown that it is capable of structural plasticity, allowing adaptation to physiological and pathological conditions [99-101]. This plasticity likely implicates kinases and phosphatases regulating the interactions between AIS components. It will be very interesting to test if these regulations, and this plasticity of the AIS structure, have an impact on the neuron organization via the regulation of protein traffic and mobility.

\section{Acknowledgements}

The authors would like to thank Kirsten Harris for the use of the AIS electron microscopy image, Francis Castets and Amapola Autillo-Touati for reading the manuscript, and the members of the Dargent lab for helpful discussions. We apologize to those colleagues whose work could not be cited because of space constraints.

\section{References}

[1] Barnes AP, Polleux F. Establishment of axon-dendrite polarity in developing neurons. Annu Rev Neurosci 2009;32:347-81.

[2] Kapitein LC, Hoogenraad CC. Which way to go? Cytoskeletal organization and polarized transport in neurons. Mol Cell Neurosci 2011;46:9-20.

[3] Rasband MN. The axon initial segment and the maintenance of neuronal polarity. Nat Rev Neurosci 2010;11:552-62.

[4] Clark BD, Goldberg EM, Rudy B. Electrogenic tuning of the axon initial segment. Neuroscientist 2009;15:651-68.

[5] Peters A, Palay SL, Webster HD. The fine structure of the nervous system. Oxford University Press: USA; 1991.

[6] Bennett V, Baines AJ. Spectrin and ankyrin-based pathways: metazoan inventions for integrating cells into tissues. Physiol Rev 2001;81:1353-92.

[7] Kordeli E, Lambert S, Bennett V, Ankyrin G. A new ankyrin gene with neuralspecific isoforms localized at the axonal initial segment and node of Ranvier. J Biol Chem 1995;270:2352-9.

[8] Iwakura A, Uchigashima M, Miyazaki T, Yamasaki M, Watanabe M. Lack of molecular-anatomical evidence for GABAergic influence on axon initial segment of cerebellar purkinje cells by the pinceau formation. J Neurosci 2012;32:9438-48

[9] Le Bras B, Fréal A, Czarnecki A, Legendre P, Bullier E, Komada M, et al. In vivo assembly of the axon initial segment in motor neurons. Brain Struct Funct 2013.

[10] Berghs S, Aggujaro D, Dirkx R, Maksimova E, Stabach P, Hermel JM, et al. BetaIV spectrin, a new spectrin localized at axon initial segments and nodes of Ranvier in the central and peripheral nervous system. J Cell Biol 2000;151:985-1002

[11] Lacas-Gervais S, Guo J, Strenzke N, Scarfone E, Kolpe M, Jahkel M, et al. BetaIVSigma1 spectrin stabilizes the nodes of Ranvier and axon initial segments. J Cell Biol 2004;166:983-90.

[12] Uemoto Y, Suzuki S-I, Terada N, Ohno N, Ohno S, Yamanaka S, et al. Specific role of the truncated betaIV-spectrin Sigma6 in sodium channel clustering at axon initial segments and nodes of Ranvier. J Biol Chem 2007;282:6548-55.

[13] Nakada C, Ritchie K, Oba Y, Nakamura M, Hotta Y, Iino R, et al. Accumulation of anchored proteins forms membrane diffusion barriers during neuronal polarization. Nat Cell Biol 2003;5:626-32.

[14] Song A-H, Wang D, Chen G, Li Y, Luo J, Duan S, et al. A selective filter for cytoplasmic transport at the axon initial segment. Cell 2009;136:1148-60.

[15] Xu K, Zhong G, Zhuang X. Actin, spectrin, and associated proteins form a periodic cytoskeletal structure in axons. Science 2013;339:452-6.

[16] Watanabe K, Al-Bassam S, Miyazaki Y, Wandless TJ, Webster P, Arnold DB. Networks of polarized actin filaments in the axon initial segment provide a mechanism for sorting axonal and dendritic proteins. Cell Rep 2012:1-13.

[17] Winckler B, Forscher P, Mellman I. A diffusion barrier maintains distribution of membrane proteins in polarized neurons. Nature 1999;397:698-701.

[18] Martin P-M, Carnaud M, Garcia del Caño G, Irondelle M, Irinopoulou T, Girault J-A, et al. Schwannomin-interacting protein-1 isoform IQCJ-SCHIP-1 is a late component of nodes of Ranvier and axon initial segments. J Neurosci 2008;28:6111-7.
[19] Srinivasan Y, Elmer L, Davis JQ, Bennett V, Angelides KJ. Ankyrin and spectrin associate with voltage-dependent sodium channels in brain. Nature 1988;333:177-80.

[20] Garrido JJ, Giraud P, Carlier E, Fernandes F, Moussif A, Fache M-P, et al. A targeting motif involved in sodium channel clustering at the axonal initial segment. Science 2003;300:2091-4.

[21] Lemaillet G, Walker B, Lambert S. Identification of a conserved ankyrinbinding motif in the family of sodium channel alpha subunits. J Biol Chem 2003;278:27333-9.

[22] Fleidervish IA, Lasser-Ross N, Gutnick MJ, Ross WN. Na ${ }^{+}$imaging reveals little difference in action potential-evoked $\mathrm{Na}^{+}$influx between axon and soma. Nat Neurosci 2010;13:852-60.

[23] Kole M, Ilschner S, Kampa B, Williams S, Ruben P, Stuart G. Action potential generation requires a high sodium channel density in the axon initial segment. Nat Neurosci 2008;11:178-86.

[24] Lorincz A, Nusser Z. Molecular identity of dendritic voltage-gated sodium channels. Science 2010;328:906-9.

[25] Kole MHP, Stuart GJ. Signal processing in the axon initial segment. Neuron 2012;73:235-47

[26] Leterrier C, Brachet A, Dargent B, Vacher H. Determinants of voltage-gated sodium channel clustering in neurons. Semin Cell Dev Biol 2011;22:171-7.

[27] Boiko T, Van Wart A, Caldwell JH, Levinson SR, Trimmer JS, Matthews G. Functional specialization of the axon initial segment by isoform-specific sodium channel targeting. J Neurosci 2003;23:2306-13.

[28] Hu W, Tian C, Li T, Yang M, Hou H, Shu Y. Distinct contributions of Na(v)1.6 and $\mathrm{Na}(\mathrm{v}) 1.2$ in action potential initiation and backpropagation. Nat Neurosci 2009;12:996-1002.

[29] Pan Z, Kao T, Horvath Z, Lemos J, Sul J-Y, Cranstoun S, et al. A common ankyrinG-based mechanism retains KCNQ and NaV channels at electrically active domains of the axon. J Neurosci 2006;26:2599-613.

[30] Ogawa Y, Horresh I, Trimmer JS, Bredt DS, Peles E, Rasband MN. Postsynaptic density-93 clusters Kv1 channels at axon initial segments independently of Caspr2. J Neurosci 2008;28:5731-9.

[31] Duflocq A, Chareyre F, Giovannini M, Couraud F, Davenne M. Characterization of the axon initial segment (AIS) of motor neurons and identification of a para-AIS and a juxtapara-AIS, organized by protein 4.1B. BMC Biol 2011;9:66

[32] Bender KJ, Trussell LO. The physiology of the axon initial segment. Annu Rev Neurosci 2012;35:249-65.

[33] Davis JQ, Lambert S, Bennett V. Molecular composition of the node of Ranvier: identification of ankyrin-binding cell adhesion molecules neurofascin (mucin+/third FNIII domain-) and NrCAM at nodal axon segments. J Cell Biol 1996;135:1355-67.

[34] Hedstrom K, Xu X, Ogawa Y, Frischknecht R, Seidenbecher C, Shrager P, et al Neurofascin assembles a specialized extracellular matrix at the axon initial segment. J Cell Biol 2007;178:875-86.

[35] Ango F, di Cristo G, Higashiyama H, Bennett V, Wu P, Huang Z. Ankyrinbased subcellular gradient of neurofascin, an immunoglobulin family protein, directs GABAergic innervation at purkinje axon initial segment. Cell 2004;119:257-72.

[36] Garver TD, Ren Q, Tuvia S, Bennett V. Tyrosine phosphorylation at a site highly conserved in the L1 family of cell adhesion molecules abolishes ankyrin binding and increases lateral mobility of neurofascin. J Cell Biol 1997;137: $703-14$.

[37] Bréchet A, Fache M-P, Brachet A, Ferracci G, Baude A, Irondelle M, et al Protein kinase CK2 contributes to the organization of sodium channels in axonal membranes by regulating their interactions with ankyrin G. J Cell Biol 2008;183:1101-14

[38] Sanchez-Ponce D, Muñoz A, Garrido JJ. Casein kinase 2 and microtubules control axon initial segment formation. Mol Cell Neurosci 2011;46: 222-34.

[39] Vacher H, Yang J-W, Cerda O, Autillo-Touati A, Dargent B, Trimmer JS. Cdk-mediated phosphorylation of the Kv $\beta 2$ auxiliary subunit regulates Kv1 channel axonal targeting. J Cell Biol 2011;192:813-24.

[40] Trunova S, Baek B, Giniger E. Cdk5 regulates the size of an axon initial segment-like compartment in mushroom body neurons of the Drosophila central brain. J Neurosci 2011;31:10451-62.

[41] Hund TJ, Koval OM, Li J, Wright PJ, Qian L, Snyder JS, et al. A B(IV)spectrin/CaMKII signaling complex is essential for membrane excitability in mice. J Clin Invest 2010;120:3508-19

[42] Tapia M, Del Puerto A, Puime A, Sanchez-Ponce D, Fronzaroli-Molinieres L Pallas-Bazarra N, et al. GSK3 and $\beta$-catenin determines functional expression of sodium channels at the axon initial segment. Cell Mol Life Sci 2012;70:105-20.

[43] Shavkunov AS, Wildburger NC, Nenov MN, James TF, Buzhdygan TP, PanovaElektronova NI, et al. The fibroblast growth factor 14 (FGF14)/voltage-gated sodium channel complex is a new target of glycogen synthase kinase 3 (GSK3). J Biol Chem 2013;288:19370-85.

[44] Davis JQ, Bennett V. Brain ankyrin. A membrane-associated protein with binding sites for spectrin, tubulin, and the cytoplasmic domain of the erythrocyte anion channel. J Biol Chem 1984;259:13550-9.

[45] Bennett V, Davis J. Erythrocyte ankyrin: immunoreactive analogues are associated with mitotic structures in cultured cells and with microtubules in brain. Proc Natl Acad Sci USA 1981;78:7550-4.

[46] Zhou D, Lambert S, Malen PL, Carpenter S, Boland LM, Bennett V. AnkyrinG is required for clustering of voltage-gated $\mathrm{Na}$ channels at axon initial segments and for normal action potential firing. J Cell Biol 1998;143:1295-304. 
[47] Sobotzik J-M, Sie JM, Politi C, Del Turco D, Bennett V, Deller T, et al. AnkyrinG is required to maintain axo-dendritic polarity in vivo. Proc Natl Acad Sci USA 2009;106:17564-9.

[48] Leterrier C, Vacher H, Fache M-P, d'Ortoli SA, Castets F, Autillo-Touati A, et al. End-binding proteins EB3 and EB1 link microtubules to ankyrin $\mathrm{G}$ in the axon initial segment. Proc Natl Acad Sci USA 2011;108:8826-31.

[49] Akhmanova A, Steinmetz MO. Microtubule+TIPs at a glance. J Cell Sci 2010;123:3415-9.

[50] Boiko T, Vakulenko M, Ewers H, Yap CCC, Norden C, Winckler B. Ankyrin-dependent and -independent mechanisms orchestrate axonal compartmentalization of L1 family members neurofascin and L1/neuron-glia cell adhesion molecule. J Neurosci 2007;27:590-603.

[51] Jenkins SM, Bennett V. Ankyrin-G coordinates assembly of the spectrin-based membrane skeleton, voltage-gated sodium channels, and L1 CAMs at Purkinje neuron initial segments. J Cell Biol 2001;155:739-46.

[52] Gasser A, Ho TS-Y, Cheng X, Chang K-J, Waxman SG, Rasband MN, et al. An ankyrinG-binding motif is necessary and sufficient for targeting Nav1.6 sodium channels to axon initial segments and nodes of Ranvier. J Neurosci 2012;32:7232-43.

[53] Rasmussen HB, Frøkjaer-Jensen C, Jensen CS, Jensen HS, Jørgensen NK, Misonou H, et al. Requirement of subunit co-assembly and ankyrin-G for Mchannel localization at the axon initial segment. J Cell Sci 2007;120:953-63.

[54] Yang Y, Ogawa Y, Hedstrom K, Rasband MN. BetaIV spectrin is recruited to axon initial segments and nodes of Ranvier by ankyring. J Cell Biol 2007:176:509-19.

[55] Xu X, Shrager P. Dependence of axon initial segment formation on $\mathrm{Na}^{+}$channel expression. J Neurosci Res 2005;79:428-41.

[56] Eshed Y, Feinberg K, Poliak S, Sabanay H, Sarig-Nadir O, Spiegel I, et al. Gliomedin mediates Schwann cell-axon interaction and the molecular assembly of the nodes of Ranvier. Neuron 2005;47:215-29.

[57] Dzhashiashvili Y, Zhang Y, Galinska J, Lam I, Grumet M, Salzer JL. Nodes of Ranvier and axon initial segments are ankyrin G-dependent domains that assemble by distinct mechanisms. J Cell Biol 2007;177:857-70.

[58] Zhang Y, Bekku Y, Dzhashiashvili Y, Armenti S, Meng X, Sasaki Y, et al. Assembly and maintenance of nodes of Ranvier rely on distinct sources of proteins and targeting mechanisms. Neuron 2012;73:92-107.

[59] Galiano MR, Jha S, Ho TS-Y, Zhang C, Ogawa Y, Chang K-J, et al. A distal axonal cytoskeleton forms an intra-axonal boundary that controls axon initial segment assembly. Cell 2012;149:1125-39.

[60] Hedstrom K, Ogawa Y, Rasband MN. AnkyrinG is required for maintenance of the axon initial segment and neuronal polarity. J Cell Biol 2008;183:635-40.

[61] Komada M, Soriano P. [Beta]IV-spectrin regulates sodium channel clustering through ankyrin-G at axon initial segments and nodes of Ranvier. J Cell Biol 2002;156:337-48.

[62] Zonta B, Desmazieres A, Rinaldi A, Tait S, Sherman DL, Nolan MF, et al. A critical role for neurofascin in regulating action potential initiation through maintenance of the axon initial segment. Neuron 2011;69:945-56.

[63] Buttermore ED, Piochon C, Wallace ML, Philpot BD, Hansel C, Bhat MA. Pinceau organization in the cerebellum requires distinct functions of neurofascin in Purkinje and basket neurons during postnatal development. J Neurosci 2012;32:4724-42.

[64] Zhang X, Bennett V. Restriction of $480 / 270-k D$ ankyrin G to axon proximal segments requires multiple ankyrin G-specific domains. J Cell Biol 1998; $142: 1571-81$

[65] He M, Tseng W-C, Bennett V. A single divergent exon inhibits ankyrin-B association with the plasma membrane. J Biol Chem 2013.

[66] He M, Jenkins P, Bennett V. Cysteine 70 of ankyrin-G is S-palmitoylated and is required for function of ankyrin-G in membrane domain assembly. J Biol Chem 2012;287:43995-4005.

[67] Garrido JJ, Fernandes F, Giraud P, Mouret I, Pasqualini E, Fache M-P, et al. Identification of an axonal determinant in the C-terminus of the sodium channel $\mathrm{Na}(\mathrm{v}) 1.2$. EMBO J 2001;20:5950-61.

[68] Fache M-P, Moussif A, Fernandes F, Giraud P, Garrido JJ, Dargent B. Endocytotic elimination and domain-selective tethering constitute a potential mechanism of protein segregation at the axonal initial segment. J Cell Biol 2004; $166: 571-8$.

[69] Chung HJ, Jan Y-N, Jan LY, Polarized axonal surface expression of neuronal KCNQ channels is mediated by multiple signals in the KCNQ2 and KCNQ3 C-terminal domains. Proc Natl Acad Sci USA 2006;103:8870-5.

[70] Yap CC, Vakulenko M, Kruczek K, Motamedi B, Digilio L, Liu JS, et al. Doublecortin (DCX) mediates endocytosis of neurofascin independently of microtubule binding. J Neurosci 2012;32:7439-53.

[71] Angelides KJ, Elmer LW, Loftus D, Elson E. Distribution and lateral mobility of voltage-dependent sodium channels in neurons. J Cell Biol 1988;106:1911-25.

[72] Brachet A, Leterrier C, Irondelle M, Fache M-P, Racine V, Sibarita J-B, et al. Ankyrin $G$ restricts ion channel diffusion at the axonal initial segment before the establishment of the diffusion barrier. J Cell Biol 2010;191:383-95.
[73] Kobayashi T, Storrie B, Simons K, Dotti CG. A functional barrier to movement of lipids in polarized neurons. Nature 1992;359:647-50.

[74] Nakata T, Hirokawa N. Microtubules provide directional cues for polarized axonal transport through interaction with kinesin motor head. J Cell Biol 2003;162:1045-55.

[75] Nishimura K, Akiyama H, Komada M, Kamiguchi H. BetaIV-spectrin forms a diffusion barrier against L1CAM at the axon initial segment. Mol Cell Neurosci 2007:34:422-30.

[76] Chan-Palay V. The tripartite structure of the undercoat in initial segments of Purkinje cell axons. Z Anat Entwicklungsgesch 1972;139:1-10.

[77] Li X, Kumar Y, Zempel H, Mandelkow E-M, Biernat J, Mandelkow E. Nove diffusion barrier for axonal retention of Tau in neurons and its failure in neurodegeneration. EMBO J 2011;30:4825-37.

[78] Burack M, Silverman M, Banker GA. The role of selective transport in neuronal protein sorting. Neuron 2000;26:465-72.

[79] Al-Bassam S, Xu M, Wandless TJ, Arnold DB. Differential trafficking of transport vesicles contributes to the localization of dendritic proteins. Cell Rep 2012;2:89-100.

[80] Lewis TL, Mao T, Svoboda K, Arnold DB. Myosin-dependent targeting of transmembrane proteins to neuronal dendrites. Nat Neurosci 2009;12:568-76.

[81] Lewis TL, Mao T, Arnold DB. A role for myosin VI in the localization of axonal proteins. PLoS Biol 2011;9:e1001021.

[82] Buffington SA, Sobotzik JM, Schultz C, Rasband MN. IкB $\alpha$ is not required for axon initial segment assembly. Mol Cell Neurosci 2012;50:1-9.

[83] Jiang K, Toedt G, Montenegro Gouveia S, Davey NE, Hua S, der Vaart van B, et al. A proteome-wide screen for mammalian SxIP motif-containing microtubule plus-end tracking proteins. Curr Biol 2012;22:1800-7

[84] Shams'ili S, de Leeuw B, Hulsenboom E, Jaarsma D, Smitt PS. A new paraneoplastic encephalomyelitis autoantibody reactive with the axon initial segment. Neurosci Lett 2009;467:169-72.

[85] Liang X, Madrid J, Gärtner R, Verbavatz J-M, Schiklenk C, Wilsch-Bräuninger $\mathrm{M}$, et al. A NOMPC-dependent membrane-microtubule connector is a candidate for the gating spring in fly mechanoreceptors. Curr Biol 2013;23: 755-63.

[86] Hirokawa N, Noda Y, Tanaka Y, Niwa S. Kinesin superfamily motor proteins and intracellular transport. Nat Rev Mol Cell Biol 2009;10:682-96.

[87] Konishi Y, Setou M. Tubulin tyrosination navigates the kinesin-1 motor domain to axons. Nat Neurosci 2009;12:559-67.

[88] Hammond JW, Huang C-F, Kaech S, Jacobson C, Banker GA, Verhey KJ. Posttranslational modifications of tubulin and the polarized transport of kinesin-1 in neurons. Mol Biol Cell 2010;21:572-83.

[89] Tapia M, Wandosell F, Garrido JJ. Impaired function of HDAC6 slows down axonal growth and interferes with axon initial segment development. PLoS ONE 2010;5:e12908.

[90] Reed NA, Cai D, Blasius TL, Jih GT, Meyhofer E, Gaertig J, et al. Microtubule acetylation promotes kinesin-1 binding and transport. Curr Biol 2006; $16: 2166-72$

[91] Dunn S, Morrison EE, Liverpool T, Molina-París C, Cross R, Alonso M, et al. Differential trafficking of Kif5c on tyrosinated and detyrosinated microtubules in live cells. J Cell Sci 2008;121:1085-95.

[92] Dimitrov A, Quesnoit M, Moutel S, Cantaloube I, Poüs C, Perez F. Detection of GTP-tubulin conformation in vivo reveals a role for GTP remnants in microtubule rescues. Science 2008;322:1353-6.

[93] Nakata T, Niwa S, Okada Y, Perez F, Hirokawa N. Preferential binding of a kinesin-1 motor to GTP-tubulin-rich microtubules underlies polarized vesicle transport. J Cell Biol 2011;194:245-55.

[94] Kapitein LC, Schlager MA, Kuijpers M, Wulf PS, van Spronsen M, MacKintosh FC, et al. Mixed microtubules steer dynein-driven cargo transport into dendrites. Curr Biol 2010;20:290-9

[95] Ye B, Zhang Y, Song W, Younger SH, Jan LY, Jan Y-N. Growing dendrites and axons differ in their reliance on the secretory pathway. Cell 2007;130: 717-29.

[96] Zheng Y, Wildonger J, Ye B, Zhang Y, Kita A, Younger SH, et al. Dynein is required for polarized dendritic transport and uniform microtubule orientation in axons. Nat Cell Biol 2008; 10:1172-80.

[97] Maniar TA, Kaplan M, Wang GJ, Shen K, Wei L, Shaw JE, et al. UNC-33 (CRMP) and ankyrin organize microtubules and localize kinesin to polarize axon-dendrite sorting. Nat Neurosci 2012;15:48-56.

[98] Edwards SL, Yu S-C, Hoover CM, Phillips BC, Richmond JE, Miller KG. An organelle gatekeeper function for Caenorhabditis elegans UNC-16 (JIP3) at the axon initial segment. Genetics 2013;194:143-61.

[99] Kuba H, Oichi Y, Ohmori H. Presynaptic activity regulates $\mathrm{Na}(+)$ channel distribution at the axon initial segment. Nature 2010;465:1075-8.

[100] Grubb MS, Burrone J. Activity-dependent relocation of the axon initial segment fine-tunes neuronal excitability. Nature 2010;465:1070-4.

[101] Schafer DP, Jha S, Liu F, Akella T, McCullough LD, Rasband MN. Disruption of the axon initial segment cytoskeleton is a new mechanism for neuronal injury. J Neurosci 2009;29:13242-54. 